\title{
A Cellular Mechanism Underlying Enhanced Capability for Complex Olfactory Discrimination Learning
}

\author{
Naveen Chandra, Richa Awasthi, Togba Ozdogan, Friedrich W. Johenning, Barbara Imbrosci, ${ }^{\circ}$ Genela \\ Morris, Dietmar Schmitz, and $\mathbb{1}^{-}$Edi Barkai
}

https://doi.org/10.1523/ENEURO.0198-18.2019

University of Haifa, Haifa 3498838 , Israel

\begin{abstract}
The biological mechanisms underlying complex forms of learning requiring the understanding of rules based on previous experience are not yet known. Previous studies have raised the intriguing possibility that improvement in complex learning tasks requires the long-term modulation of intrinsic neuronal excitability, induced by reducing the conductance of the slow calcium-dependent potassium current $\left(\mathrm{sl}_{\mathrm{AHP}}\right)$ simultaneously in most neurons in the relevant neuronal networks in several key brain areas. Such $\mathrm{sl}_{\mathrm{AHP}}$ reduction is expressed in attenuation of the postburst afterhyperpolarization (AHP) potential, and thus in enhanced repetitive action potential firing. Using complex olfactory discrimination (OD) learning as a model for complex learning, we show that brief activation of the GluK2 subtype glutamate receptor results in long-lasting enhancement of neuronal excitability in neurons from controls, but not from trained rats. Such an effect can be obtained by a brief tetanic synaptic stimulation or by direct application of kainate, both of which reduce the postburst AHP in pyramidal neurons. Induction of long-lasting enhancement of neuronal excitability is mediated via a metabotropic process that requires PKC and ERK activation. Intrinsic neuronal excitability cannot be modulated by synaptic activation in neurons from GluK2 knock-out mice. Accordingly, these mice are incapable of learning the complex OD task. Moreover, viral-induced overexpression of Gluk2 in piriform cortex pyramidal neurons results in remarkable enhancement of complex OD learning. Thus, signaling via kainate receptors has a central functional role in higher cognitive abilities.
\end{abstract}

Key words: GIUk2 receptors; intracellular recordings; meta-learning; olfactory-learning; pyramidal neurons; spost-burst AHP

\section{Significance Statement}

In this study, we describe the molecular and cellular mechanism for complex learning. Previous studies suggest that complex learning is accompanied by enhanced intrinsic neuronal excitability, induced by reducing the conductance of a slow potassium current in most neurons in the relevant brain areas. Using complex olfactory discrimination learning, we show that the kainate GluK2 subtype glutamate receptor is necessary and sufficient for complex learning. Intrinsic neuronal excitability cannot be modulated in neurons from GluK2 knock-out mice. Accordingly, these mice are incapable of learning the complex task. Moreover, viral-induced overexpression of Gluk2 in piriform cortex pyramidal neurons enhances complex learning remarkably. Thus, postsynaptic activation of GluK2 receptors has a central functional role in higher cognitive abilities.

Received May 21, 2018; accepted January 6, 2019; First published January 31, 2019.

The authors declare no competing financial interests.
Author contributions: D.S. and E.B. designed research; N.C., R.A., T.O., F.W.J., B.I., and G.M. performed research; N.C., R.A., T.O., F.W.J., and G.M. analyzed data; D.S. and E.B. wrote the paper. 


\section{Introduction}

Training rodents in a complex olfactory discrimination (OD) task results in enhanced performance over repeated training sessions, as they need to understand and apply a set of complex rules (Saar and Barkai 2003; Zelcer et al., 2006; Chandra and Barkai, 2018). Since such high-order and complex learning would presumably require the active participation and modulation of multiple brain areas and neuronal networks, it was suggested to be mediated by the combined action of large groups of neurons (Hasselmo and Stern, 2018). At the cellular and synaptic level, the simultaneous modulation of many synaptic connections and neurons has been suggested to occur via a process termed metaplasticity. Metaplasticity is defined as a persistent and activity-dependent change in the functional state of the neuron that affects future synaptic plasticity (Abraham and Bear, 1996; Abraham, 2008; Hulme et al., 2013). Notably, it is yet to be shown that metaplasticity mechanisms that could prepare neuronal networks to encode specific content indeed underlie complex learning on the behavioral level (Hulme et al., 2013).

In this context, learning induced enhancement in intrinsic neuronal excitability is a global phenomenon observed in a multitude of brain regions and behavioral tasks (Moyer et al., 1996; Thompson et al., 1996; Saar et al., 2001; Oh et al., 2003; Zelcer et al., 2006; Motanis et al., 2014). In particular, for the olfactory discrimination learning paradigm central to this study, global changes in neuronal intrinsic excitability occur in different brain regions, specifically the piriform cortex (Saar et al., 1998, 2001), the hippocampal CA1 area (Zelcer et al., 2006), and the basolateral amygdala (Motanis et al., 2014).

Spike frequency adaptation is an important regulatory mechanism for intrinsic excitability. In hippocampal and cortical pyramidal neurons, spike frequency adaptation is modulated by medium and slow afterhyperpolarizations (AHPs), generated by calcium-dependent hyperpolarizing potassium currents (Madison and Nicoll, 1984; Constanti and Sim, 1987). Indeed, learning-induced long-term reduction in the postburst AHP and the resulting enhanced excitability is mediated by a long-term decrease in the conductance of the slow calcium-dependent potassium current ( $\mathrm{sl}_{\mathrm{AHP}}$ ), in both the piriform cortex and hippocampus (Power et al., 2002; Brosh et al., 2006). At the molecular level, learning-induced long-term AHP reduction depends on persistent activation of PKC and ERK second-messenger systems (CohenMatsliah et al., 2007). It is as yet unclear how AHP-mediated intrinsic excitability modifications are induced during learning.

This research was supported by the Israel Academy of Sciences and Humanities (Grant 501100003973); Deutsche Forschungsgemeinschaft (Grant 501100001659); and Bundesministerium Bildung und Forschung (Grant 501100002347).

Correspondence should be addressed to Edi Barkai at ebarkai@ research.haifa.ac.il.

https://doi.org/10.1523/ENEURO.0198-18.2019

Copyright @ 2019 Chandra et al.

This is an open-access article distributed under the terms of the Creative Commons Attribution 4.0 International license, which permits unrestricted use, distribution and reproduction in any medium provided that the original work is properly attributed.
Within the hippocampal formation, the GluK2 subtype glutamate receptor enhances intrinsic neuronal excitability via noncanonical signaling (Fisahn et al., 2005; Melyan and Wheal, 2011, Chamberlain et al., 2013). Such synaptic activity-induced and kainate-induced AHP reduction is also dependent on PKC and ERK activation (Melyan et al., 2002, 2004; Grabauskas et al., 2007). This noncanonical GluK2 function is distinct from its direct ionotropic action on synaptic transmission (Ruiz et al., 2005). Interestingly, humans lacking the gene for GluK2 suffer from intellectual disability (Motazacker et al., 2007). To date, the noncanonical signaling of GluK2 receptors has been related to neuronal plasticity in terms of long-term potentiation (LTP) only (Petrovic et al., 2017).

Here, we show that signaling via the GluK2 subtype receptor has a key role in complex OD learning. In vitro induced long-term AHP reduction is occluded by prior learning-induced AHP reduction. Moreover, GluK2 activity is both necessary and sufficient for the enhancement of complex learning capabilities. Our data suggest that this glutamatergic receptor and the downstream metaplastic AHP reduction have a central role in complex learning.

\section{Materials and Methods}

\section{Animal training}

Rat training in complex olfactory learning

Subjects and apparatus. Age-matched young adult Sprague Dawley male rats were maintained on a $23.5 \mathrm{~h}$ water-deprivation schedule before training, with food available ad libitum. Olfactory discrimination training protocol was performed daily on each trained and pseudotrained rat in a four-arm radial maze, as previously described (Saar et al., 1998, 2001), with commercial synthetic odors that are regularly used in the cosmetics and food industry purchased from Value Fragrances \& Flavors. Odors were diluted by factor of 1000 before use in the olfactory maze. Odors were composed from natural and naturally derived materials in their formulations. Odors used in this study were as follows: apple, skin musk, and lavender.

Training. Olfactory training consisted of 20 trials per day for each rat, as previously described (Saar et al., 1998). In short, in each trial the rat had to choose between two odors (positive cue and negative cue) presented simultaneously. Rats designated to the trained group were rewarded on choosing the positive cue. Rats in the pseudotrained group were rewarded in a random fashion on choosing any odor. The criterion for learning was at least $80 \%$ positive-cue choices in the last 10 trials of a training day, as was previously used (Saar et al., 1998; 2001). Rats in the naive group were water restricted, but not exposed to the maze. Typically, two to three trained rats and two to three pseudotrained rats were trained at the same training period. As previously described (Saar et al., 1998, 2001), normal rats required $7-8 \mathrm{~d}$ of training for achieving the criterion for learning.

\section{Mice training in the complex olfactory learning}

Subjects and apparatus. GluK2 $2^{-/-}$mice on a C57BL/6J background and their wild-type littermates were trained in an olfactory maze similar to that used for rats, with few 
adjustments to better fit mice. The arms of the maze were enclosed by $20-\mathrm{cm}$-high walls, and each running track was $10 \mathrm{~cm}$ in width and $35 \mathrm{~cm}$ in length. Entries to arms to were blocked by doors, which opened at the beginning of each trial. The center area of the maze contained four odor pokes in front of each track. The end of each track contained a drinking well ( $1.5 \mathrm{~cm}$ radius), which received water by means of an automated valve. The maze was controlled by a computer, which sent transistor-transistor logic pulses at the beginning of each trial to cue the active odor pokes, to open the valves to release odors into the pokes, and to activate the valve that pumps water into the drinking wells.

Training. Before the start of experiments, each animal was handled for $5 \mathrm{~d}$ and was habituated to the training arena for $3 \mathrm{~d}$. The water restriction protocol started $23 \mathrm{~h}$ before the first training day. Animals were allowed to drink water daily ad libitum in their home cage for $1 \mathrm{~h}$ and 15 min after the training.

Each training session consisted of 20 trials. At the beginning of each trial, two odor pokes were illuminated to cue the odor pokes that were active for that trial, and two different odors were presented (varied across different batches of mice), one of which was associated with water reward. If the animal chose to enter the correct track, it received $0.2 \mathrm{ml}$ of water at the end of the track. In case of wrong entry, the animal did not receive any water and walked back to the center of the maze and waited for the next trial to start. Experiments aimed to examine the effect of GluK2 overexpression on complex olfactory learning were performed blind.

\section{Simple olfactory task (cookie test)}

After the completion of training in the complex olfactory plus maze, the mice were tested for basic olfactory function using the buried food or cookie test. Mice were habituated to butter cookies for $2 \mathrm{~d}$ and were subsequently slightly food restricted for $8 \mathrm{~h}$ before the cookie test. Each mouse started the test in a $20 \times 40 \mathrm{~cm}$ cage (identical in shape and size to their home cage) and freely foraged for the cookie, which was buried in the cage bedding. We used latency to find the cookie as the parameter for the cookie test learning.

\section{Electrophysiology}

\section{Sharp electrode recordings}

The $400 \mu \mathrm{m}$ coronal piriform cortex rat brain slices were cut as previously described (Saar et al., 1998) and were kept in oxygenated $\left(95 \% \mathrm{O}_{2}+5 \% \mathrm{CO}_{2}\right)$ normal saline Ringer's solution as follows (in $\mathrm{mm}$ ): $\mathrm{NaCl} 124, \mathrm{KCl} 3$, $\mathrm{MgSO}_{4} 2, \mathrm{NaH}_{2} \mathrm{PO}_{4} 1.25, \mathrm{NaHCO}_{3} 26, \mathrm{CaCl}_{2} 2$, and glucose 10. Intracellular recordings were obtained from pyramidal cells in layer II of the piriform cortex, with $4 \mathrm{M}$ $\mathrm{K}$-acetate-filled sharp glass microelectrodes at $35^{\circ} \mathrm{C}$. Several piriform cortex slices were obtained from each rat. Slices were placed in a recording chamber and perfused with Ringer's solution. Intracellular recordings with sharp electrodes were obtained as previously described (Cohen-Matsliah et al., 2007). Recordings were performed using Axopatch 1D (Molecular Devices), and the data were acquired using pClamp9 (Molecular Devices). All experiments were performed blind; the identity of the rat from which neurons were recorded (naive, trained, or pseudotrained) was not known to the person conducting the experiments and measurements. One to three neurons were recorded from each rat. AHPs were recorded within minutes after good recording conditions were established [resting potential of at least $-65 \mathrm{mV}$ and action potential (AP) amplitude of $\geq 80 \mathrm{mV}$. To standardize AHP recordings, neurons were depolarized to holding potential of $-60 \mathrm{mV}$ by direct current application via the recording electrode. Postburst AHP amplitude was then measured following a $100 \mathrm{~ms}$ depolarizing current step with an intensity that generates six action potentials (Fig. 1C2 ,D2). The AHP amplitude was evoked in responses to intrinsic stimuli applied once every $10 \mathrm{~s}$. The postburst AHP amplitude was measured from baseline to the peak of the hyperpolarizing voltage deflection that follows an evoked train of six action potentials. The stimulus intensity required to evoke six action potentials always remained constant throughout the recording period of each neuron, before and after drug application. Such stability of the required stimulus intensity well coincides with the stability of basic membrane properties, such as the input resistance or resting potential of the neurons, which were not affected by the drugs. Our previous study showed that the AHP amplitudes are not modified in neurons from pseudotrained rats, compared with neurons from naive rats (Saar et al., 1998; Brosh et al., 2006; Cohen-Matsliah et al., 2007; Saar et al., 2012). Therefore, neurons from naive and pseudotrained rats were pooled to form the control group. Synaptic stimulation was delivered via a bipolar tungsten electrode, positioned at layer lb, to activate the intracortical axons, which interconnect the recorded pyramidal neurons (Fig. 1B; Saar et al., 2002) Stimulus intensity was adjusted to evoke PSPs with amplitudes of $10 \mathrm{mV}$ at a membrane potential of $-80 \mathrm{mV}$. Activity-induced AHP reduction was induced by applying 20 repetitive stimuli at $50 \mathrm{~Hz}$.

Drugs were applied into the perfusing Ringer's solution at the following concentrations: kainite, 200 nm; ERK inhibitor UO126, $30 \mu \mathrm{M}$; PKC activator 1-oleoyl-20acety-snglycerol (OAG), $10 \mu \mathrm{M}$. Slices were exposed to drugs for at least 20 min before the recordings. All cells were recorded before and after drug application.

\section{Whole-cell patch-clamp recordings}

Male C57BL/6 mice, 4-6 weeks of age, were decapitated following isoflurane anesthesia. Brains were transferred to ice-cold artificial CSF (ACSF) slicing solution containing the following (in $\mathrm{mm}$ ): $87 \mathrm{NaCl}, 50$ sucrose, 26 $\mathrm{NaHCO}_{3}, 10$ glucose, $2.5 \mathrm{KCl}, 1.25 \mathrm{NaH}_{2} \mathrm{PO}_{4}, 3 \mathrm{MgCl}_{2}$, and $0.5 \mathrm{CaCl}_{2}, \mathrm{pH}$ 7.4. Coronal slices from the piriform cortex (400 $\mu \mathrm{m}$ thickness) were cut on a slicer (model VT1200S, Leica) and stored at $32-34^{\circ} \mathrm{C}$ in the same ACSF slicing solution for $30 \mathrm{~min}$. Subsequently, slices were transferred in a standard ASCF containing (in mM) 119 $\mathrm{NaCl}, 26 \mathrm{NaHCO}_{3}, 10$ glucose, $2.5 \mathrm{KCl}, 1.0 \mathrm{NaH}_{2} \mathrm{PO}_{4}, 1.3$ $\mathrm{MgCl}_{2}$, and $2.5 \mathrm{CaCl}_{2}$, and were allowed to recover for an additional $30 \mathrm{~min}$ at room temperature. The ACSF was equilibrated with carbogen $\left(95 \% \mathrm{O}_{2}, 5 \% \mathrm{CO}_{2}\right)$. Recordings were performed in the standard ACSF at $31-32^{\circ} \mathrm{C}$ in a submerged-type recording chamber perfused at a high 
A

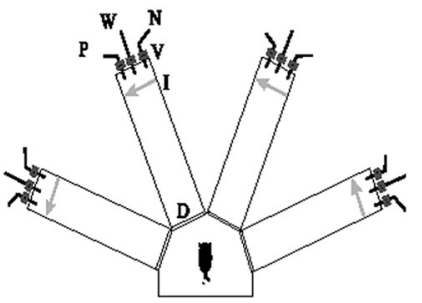

$C_{1}$

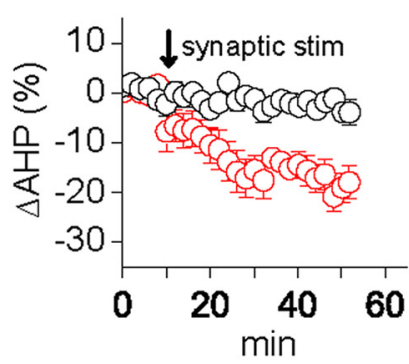

$D_{1}$

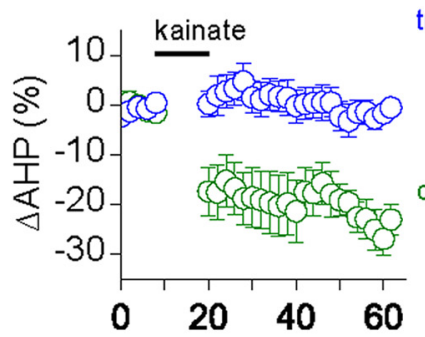

$\mathrm{D}_{2}$

trained
B
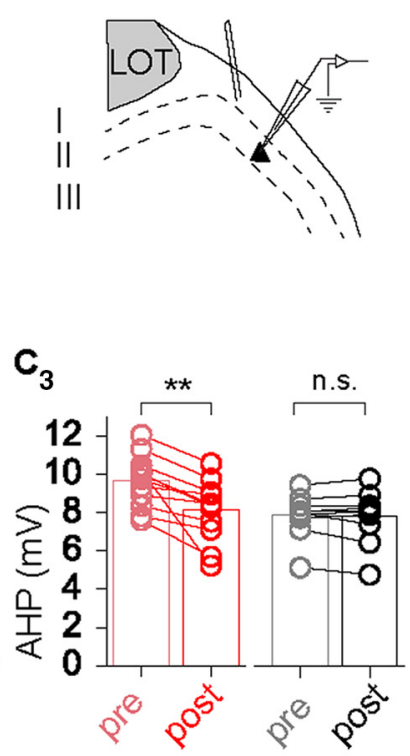

$10 \mathrm{mV}$

$100 \mathrm{~ms}$

$\mathrm{D}_{3}$

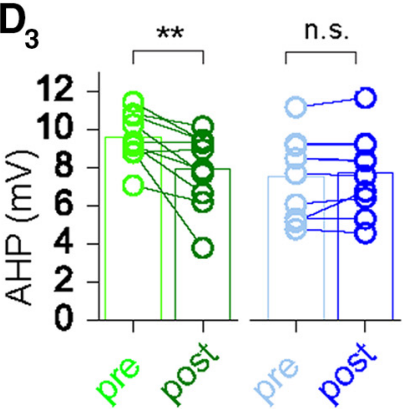

Figure 1. Learning occludes repetitive synaptic stimulation-induced and kainate-induced AHP reduction. $\boldsymbol{A}$, Schematic description of the four-arm maze. Protocols for trained and pseudotrained rats are similar: an electronic "start" command randomly opens two of eight valves $(\mathrm{V})$, releasing a positive-cue odor $(\mathrm{P})$ into one of the arms and a negative-cue odor $(\mathrm{N})$ into another. Eight seconds later, the two corresponding guillotine doors (D) are lifted to allow the rat to enter the selected arms. On reaching the far end of an arm (90 $\mathrm{cm}$ long), the rat body interrupts an infrared beam (l; arrow) and a drop of drinking water is released from a water hose (W) into a small drinking well (trained rats: only if the arm contains the positive-cue odor, pseudotrained rats: random assignment of odors to rewards). $\boldsymbol{B}$, Position of the intracellular recording electrode in layer II of the anterior piriform cortex in acute coronal brain slices and the stimulation electrode in layer lb. $\boldsymbol{C}_{1}$, Time line of long-term AHP reduction induced by tetanic synaptic stimulation of trained (black) and control (red) rats. $\boldsymbol{C}_{2}$, Averaged typical traces of a neuron from a trained (black) and pseudotrained (red) rat before (light shade) and after (dark shade) synaptic stimulation. The AP peaks are clipped to facilitate comparison of the postburst AHP. $\boldsymbol{C}_{3}$, Direct comparison of the absolute AHP amplitudes before and 20 min after synaptic stimulation for control (red) and trained (black) rats. $\boldsymbol{D}_{\mathbf{1}}$, Time line of long-term AHP reduction induced by kainate application to brain slices of trained (blue) and control (green) rats. $\boldsymbol{D}_{\mathbf{2}}$, Averaged typical traces of a neuron from a trained (blue) and pseudotrained (green) rat before (light shade) and after (dark shade) kainate application. The AP peaks are clipped to facilitate comparison of the postburst AHP. $\boldsymbol{D}_{3}$, Direct comparison of the absolute AHP amplitudes before and 20 min after kainate application for pseudotrained (green) and trained (blue) rats. Values are represented as the mean \pm SEM. ${ }^{* *} p<0.01$.

rate $(5-6 \mathrm{ml} / \mathrm{min})$. Data were collected using a Multiclamp $700 \mathrm{~A}$ amplifier (Molecular Devices) and sampled at 20 $\mathrm{KHz}$. Whole-cell recordings were performed from layer II pyramidal cells with borosilicate glass electrodes (2-5 $\mathrm{M} \Omega$ ) filled with the following (in $\mathrm{mM}$ ): $120 \mathrm{~K}$-gluconate, 10 HEPES, $10 \mathrm{KCl}, 3 \mathrm{Mg}$-ATP, 5 EGTA, $2 \mathrm{MgSO}_{4}, 0.3 \mathrm{Na}-$ GTP, and 14 phosphocreatine. The $\mathrm{pH}$ was adjusted to 7.4 with $\mathrm{KOH}$. Neurons were recorded in voltage-clamp mode at $-70 \mathrm{mV}$ in the presence of the NMDA receptor blocker,

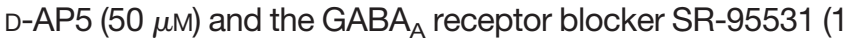
$\mu \mathrm{M})$. A single extracellular stimulation pulse was delivered every $15 \mathrm{~s}$ to evoke postsynaptic currents in the recorded neurons. After achieving a stable baseline, the selective AMPA receptor blocker GYKI-53655 $(100 \mu \mathrm{M})$ was applied to investigate the potential presence of kainate receptormediated currents. In addition, to exclude the presence of extrasynaptic kainate receptors, a train of five stimuli at 200 $\mathrm{Hz}$ was delivered under the same recording conditions.

\section{Statistical analysis}

For behavioral experiments, $t$ tests were used for statistical comparison between two groups. The effect of repetitive stimulation and drug application was examined for each neuron with and without the treatment, using a 
Table 1: Summary of the nine experimental groups

\begin{tabular}{lll}
\hline Naive-control & Naive-GFP only & Naive-GluK2 \\
Trained-control & Trained-GFP only & Trained-GluK2 \\
Pseudo-control & Pseudo-GFP only & Pseudo-GluK2
\end{tabular}

paired $t$ test. Values throughout the text and in graphs are presented as the mean \pm SE.

\section{Viral injections}

\section{Apparatus and surgery}

Animals were blurred with isoflurane and then anesthetized by the injection of ketamine $10 \%(0.09 \mathrm{cc} / 100 \mathrm{~g})$ and Dormitor $(0.05 \mathrm{cc} / 100 \mathrm{~g})$. Before the surgery, rats received treatment with an antibiotic (amoxicillin veterinary, $0.2 \mathrm{ml}$ ) and a painkiller (Calmagin, $0.03 \mathrm{ml} / 100 \mathrm{~g}$ ). Additional anesthesia ( $1 \%$ isoflurane in $\mathrm{O}_{2}$, by inhalation) was supplied as needed. Aseptic surgeries were performed on a commercial stereotaxic device (David Kopf), using a surgical microscope (Zeiss). Physiologic vital conditions were monitored throughout the surgery, and postoperative care was provided to minimize pain and ensure a healthy recovery. Small holes were drilled in the skull to allow the insertion of the syringe in the brain. The locations of the holes were determined according to atlas with bregma as the reference point. A quartz micropipette filled with $200 \mathrm{nl}$ of high-titer lentivirus stock was directed to layer II of the anterior piriform cortex. Virus was pressure injected using a Picospritzer (General Valve). The virus was injected bilaterally. The coordinates in the pirform cortex area were as follows: anteroposterior, +1.3; $\mathrm{ML}, \pm 3.5 ; \mathrm{DV},-8$.

The surgery took place in a lentivirus special room. To ensure virus expression and to allow rats to recover from the surgery, the behavioral task began $10 \mathrm{~d}$ following surgery. The procedure was performed in strict accordance with local university regulations and the guidelines of the National Institutes of Health. Following injection, animals were maintained in a warmed cage under inspection for at least $2 \mathrm{~h}$ for recovery and then placed in a designated area in the animal facility.

Animals were divided into the following three groups: (1) rats not injected with virus; (2) rats injected with a control GFP virus; and (3) rats injected with a Gluk2 virus. Each of these groups will then be further divided into the following three behavioral groups: naive, trained, and pseudotrained (Table 1). Every cage contained one rat. Rats were kept under $12 \mathrm{~h}$ light/dark cycle conditions.

\section{Virus preparation}

We used the HIV-1 Lentiviral Vector System (Ramezani and Hawley, 2002) and a pUbiquitin-IRES-GFP plasmid (based on FU-mKate2-mem-Cer-SV2a-Wm2 $13.024 \mathrm{~kb}$ ). We exchanged the pUbiquitin promoter with CaMK2a promoter (thus allowing the virus to infect only the excitatory pyramidal neurons) and inserted the cDNA of $\mathrm{GLU}_{\mathrm{RG}}$. Our final virus had the following composition:

5'CaMKIlaGluK2IRESGFP3'

\section{Recovery from virus injection}

As noted above, virus injection was followed by a $10 \mathrm{~d}$ resting period, after which olfactory discrimination training was performed in the complex OD task until rule learning was obtained. Notably, it has been shown that kainiteinduced epileptic activity (for review, see Louboutin and Strayer, 2013). Specifically, GluK2 overexpression has been shown to produce seizures (Telfeian et al., 2000). On the other hand, it has been proposed that ketogenic diet-induced upregulation of GluK2 may prevent epileptogenesis by the inhibition of excitatory synaptic transmission at specific hippocampal pathways ( $\mathrm{Xu}$ et al., 2008). To examine whether GluK2 overexpression results in epileptic events, rats were closely watched during the recovery period. None of the GluK2 virus-injected rats showed abnormal motor behavior.

\section{Immunohistochemistry}

Free-floating 50-mm-thick brain sections were collected in a cryostat (Leica) in PBS azide $0.05 \%$ and stored at $4^{\circ} \mathrm{C}$ until further use. Blocking was performed in $3 \%$ BSA with $0.3 \%$ Triton $\mathrm{X}-100$ for $1-1.5 \mathrm{~h}$ at room temperature. Sections were incubated overnight with anti-GFP primary antibody at $4^{\circ} \mathrm{C}(1: 1000$ in PBS; anti-GFP IgG fraction; Thermo Fisher Scientific). Sections were then rinsed 4 times for 10 min each, with PBS incubated with the secondary antibody (1:2000 in PBS; goat anti-rabbit IgG; Thermo Fisher Scientific) for $2 \mathrm{~h}$ at room temperature. Sections were then washed twice for $10 \mathrm{~min}$ each and then were mounted on slides using (Fluoromount Aqueous Mounting Medium, Sigma-Aldrich).

\section{Results}

\section{Synaptic stimulation induces long-term reduction of} the postburst AHP in neurons from control rats only

To test whether complex OD learning and synaptically mediated AHP reduction share common pathways, we compared AHP reduction by tetanic synaptic stimulation in the piriform cortex of acute brain slices from animals trained in a complex olfactory discrimination paradigm and controls (naive or pseudotrained; see Materials and Methods; Fig. 1A). Trained rats learned how to discriminate between the two odors and chose the arm of the maze from which the rewarded odor originated. In the pseudotrained group, the reward was randomly assigned to one of the two odors in different trials. Naïve rats were not exposed to the maze or odors.

The postburst AHP in neurons from trained rats was not affected by a weak tetanus $(20$ pulses at $50 \mathrm{~Hz})$. The averaged AHP before $(7.9 \pm 0.4 \mathrm{mV} ; n=10)$ and after $(7.8$ $\pm 0.4 \mathrm{mV} ; n=10)$ the repetitive stimuli was similar $(p=$ 0.62 , paired $t$ test; Fig. $1 C$ ). In neurons taken from control rats, repetitive synaptic stimulation significantly reduced the AHP in piriform cortex pyramidal neurons (before stimulation, $9.7 \pm 0.4 \mathrm{mV}$; after stimulation, $8.1 \pm 0.4 \mathrm{mV}$; $n=12 ; p<0.01$, paired $t$ test; Fig. 1C). Consequently, while the averaged AHP amplitude in neurons from trained rats was significantly lower than that in neurons from control rats $(p<0.01$; Saar et al., 1998, 2001; Cohen-Matsliah et al., 2007; Chandra and Barkai, 2018) following tetanic synaptic stimulation, the difference between the averaged values was abolished $(p=0.64)$. Similar to what was previously reported in the hippocampus (Melyan et al., 2004), the tetanus-induced AHP reduc- 

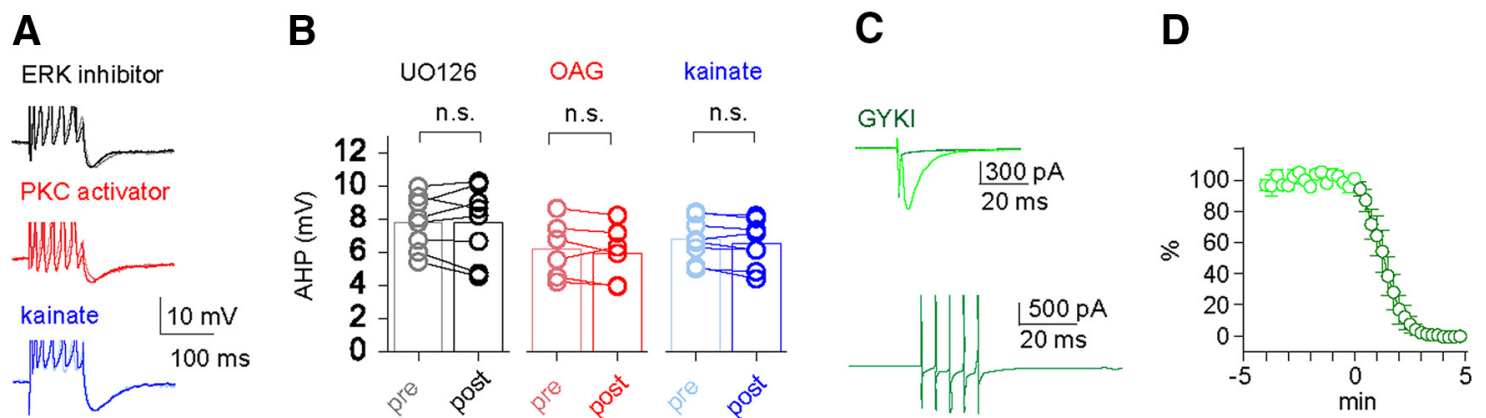

Figure 2. Activity-induced AHP reduction is mediated by intrinsic activity of second-messenger systems, and not by synaptic activation of kainate receptors. $\boldsymbol{A}$, Averaged typical traces in three neurons taken from naive rats before (light shade) and after (dark shade) repetitive synaptic stimulation. The AP peaks are clipped to facilitate comparison of the postburst AHP. Repetitive synaptic stimulation-induced post-AHP reduction is abolished by prior application of the ERK inhibitor UO126 (top traces), prior application of the PKC activator OAG (middle traces), or by prior kainate application (bottom traces). $\boldsymbol{B}$, Direct comparison of the absolute AHP amplitudes before and $20 \mathrm{~min}$ after repetitive synaptic stimulation in the presence UO126 (black), OAG (red), or kainate (blue). Each of the three treatments blocked the synaptic activity-induced AHP reduction. Note that the agonists kainate and OAG reduced the averaged AHP in naive neurons to the averaged value observed in neurons from trained rats, while the antagonist OU126 had no direct effect on the AHP. Values represent the mean \pm SEM. C, Synaptic currents evoked by stimulating the intrinsic fibers pathway (layer lb) do not contain a kainate receptor-mediated component. The application of GYKI53655 completely blocked the synaptic currents (top trace). The bottom trace shows the lack of response to a train of five stimuli at $200 \mathrm{~Hz}$ in the same cells after GYKI53655 application. $\boldsymbol{D}$, Time line of the synaptic current amplitude before and after GYKI53655 application. Recordings were obtained in six pyramidal cells taken from three mice. Notably, the synaptic current amplitude is abolished in all recorded neurons. Values represent the mean \pm SEM.

tion remained stable for the total recording period, up to 40 min after induction (Fig. 1C). The tetanus we used (20 stimuli at $50 \mathrm{~Hz}$ ) is substantially milder than that required to elicit GluK2-dependent LTP (Petrovic et al., 2017).

\section{Kainate application reduces the AHP in neurons from control rats only}

In hippocampus, the AHP reduction by tetanic stimulation is mediated by kainate receptors (Melyan et al., 2004). In piriform cortex of control rats, the application of $200 \mathrm{~nm}$ kainate significantly reduced the AHP (before stimulation, $9.6 \pm 0.4 \mathrm{mV}$; after stimulation, $7.9 \pm 0.6 \mathrm{mV} ; n=10 ; p<$ 0.01 , paired $t$ test; Fig. $1 D$ ). This decrease outlasted the kainate washin and was stable for the total length of the recording (Fig. 1D).

In trained rats, after learning the complex olfactory discrimination paradigm, kainate application did not affect the AHP (before stimulation, $7.6 \pm 0.7 \mathrm{mV}$; after stimulation, $7.8 \pm 0.7 \mathrm{mV} ; n=10 ; p=0.29$, paired $t$ test; Fig. $1 D$ ). Thus, here too the averaged AHP amplitude in neurons from trained rats was significantly lower than in neurons from control rats $(p<0.05)$ before kainate application, and the difference between the averaged values was abolished following drug application $(p=0.84)$. We conclude that the AHP reduction resulting from complex olfactory discrimination learning mechanistically occludes AHP reduction both by tetanic stimulation and by nanomolar kainate application.

\section{Activity-induced AHP reduction is dependent on ERK and PKC activation}

We then tested whether repetitive stimulation-induced, kainate-induced, and learning-induced long-lasting AHP reduction share common second-messenger systems. To this end, we performed three sets of complementary experiments.
In the first experiment, we activated PKC. We previously showed that such persistent PKC activation is required for the long-term maintenance of AHP reduction (Seroussi et al., 2002; Cohen-Matsliah et al., 2007). We examined whether such persistent PKC phosphorylation, that results in long-term AHP reduction, prevents further AHP reduction by repetitive synaptic activation. As previously reported, in the presence of the PKC activator OAG, the amplitude of the AHP in neurons from naive rats is significantly lower than that recorded in control conditions, and resembles that observed in neurons from trained rats (Seroussi et al., 2002). In these conditions, tetanic stimulation did not further reduce the AHP in pyramidal neurons. The averaged AHP value measured in six neurons was $6.16 \pm 0.7$ before stimulation and $5.90 \pm$ 0.7 after stimulation, indicating that once the AHP is reduced by PKC activation, repetitive synaptic stimulation has no further effect on its amplitude (Fig. $2 A, B$ ).

In the second experiment, we blocked ERK activation to examine whether interrupting the phosphorylation sequence that is required for long-term maintenance learning-induced AHP reduction (Cohen-Matsliah et al., 2007) would also prevent activity-dependent AHP reduction. Indeed, in the presence of the ERK inhibitor U0126, repetitive synaptic stimulation did not induce any reduction in the AHP amplitude. The averaged AHP value measured in eight neurons was $7.78 \pm 0.6$ before stimulation and $7.76 \pm 0.8$ after stimulation (Fig. $2 A, B$ ).

In the third experiment, we examined the effect of repetitive stimulation on the AHP after kainate application. The averaged AHP value measured in eight neurons was $6.76 \pm 0.5$ before stimulation and $6.52 \pm 0.5$ after stimulation, indicating that kainate application occludes further AHP reduction by repetitive synaptic activation (Fig. $2 A, B)$. 
Notably, synaptic stimulation, both single as well as repetitive, did not evoke any measurable kainite receptormediated EPSCs (Fig. 2C,D).

Synaptic currents were evoked by stimulation in the presence of the NMDA receptor blocker, D-AP5 (50 $\mu \mathrm{M})$ and the $\mathrm{GABA}_{\mathrm{A}}$ receptor blocker gabazine $(1 \mu \mathrm{M})$. The synaptic current evoked in this condition was completely abolished by the application of tGYKI53655 (100 $\mu \mathrm{M})$, a selective AMPA receptor antagonist (Fig. $2 C, D$ ). This concentration was chosen based on the original description of synaptically activated kainite receptors on CA3 pyramidal neurons in brain slices (Castillo et al, 1997; Vignes and Collingridge, 1997). There, the authors used $100 \mu \mathrm{M}$ GYKI and accepted a partial block of kainite receptors, but yielded a full antagonism of AMPA receptors. We submit that, although we partially antagonize kainite receptors, we would still be able detect a significant expression of these receptors if expressed/anchored at synapses. Thus, synaptic currents evoked in this pathway are not mediated by kainate receptors. The downstream effects of kainate receptor activation are therefore not ionotropic but metabotropic.

\section{GluK2 mediates excitability change and complex OD learning}

In the hippocampus, the activity-induced AHP reduction is GluK2 dependent (Fisahn et al., 2005). We therefore compared the effect of nanomolar kainate on piriform cortex layer II pyramidal neurons from wild-type mice and GluK2 knockouts. In neurons from wild types, kainate application (200 nM) reduced the AHP amplitude from 7.5 \pm 0.8 to $6.5 \pm 0.9 \mathrm{mV}(n=7 ; p<0.05$, paired $t$ test; Fig. $3 A)$. In contrast, kainate did not have an effect on the AHP in neurons from GluK2 knockouts (before stimulation, 7.6 $\pm 0.7 \mathrm{mV}$; after stimulation, $7.8 \pm 0.7 \mathrm{mV} ; n=10 ; p=$ 0.29 , paired $t$ test; Fig. $3 A$ ). Together, these data strongly indicate that GluK2 activation induces the long-term learning-induced AHP reduction, a strong cellular correlate of enhancement in learning capabilities (Saar et al., 1998; Zelcer et al., 2006; Chandra and Barkai, 2018).

Next, we examined the effect of GluK2 knockout on complex olfactory learning. Although the complex olfactory discrimination task was initially developed and used for training rats, wild-type mice are also capable of learning the task, but they require more time to reach the criteria for successful completion of complex OD learning (Fig. 3B). In sharp contrast, GluK2 knock-out mice are essentially incapable of completing the complex learning task (Fig. 3B).

To ensure that the GluK2 knock-out mice did not differ from the wild-type mice in general olfactory ability or motivational state, we performed a buried food test (also called cookie test; Yang and Crawley, 2009). In this paradigm, an appetitive and familiar food reward (butter cookie) is buried in the bedding of a clean cage. Animals are compared with respect to their ability to find the buried food and with the corresponding latencies. GluK2 knockouts did not differ from wild-type mice in the time they spent exploring the new environment until they found the cookie they had been previously habituated to (la-
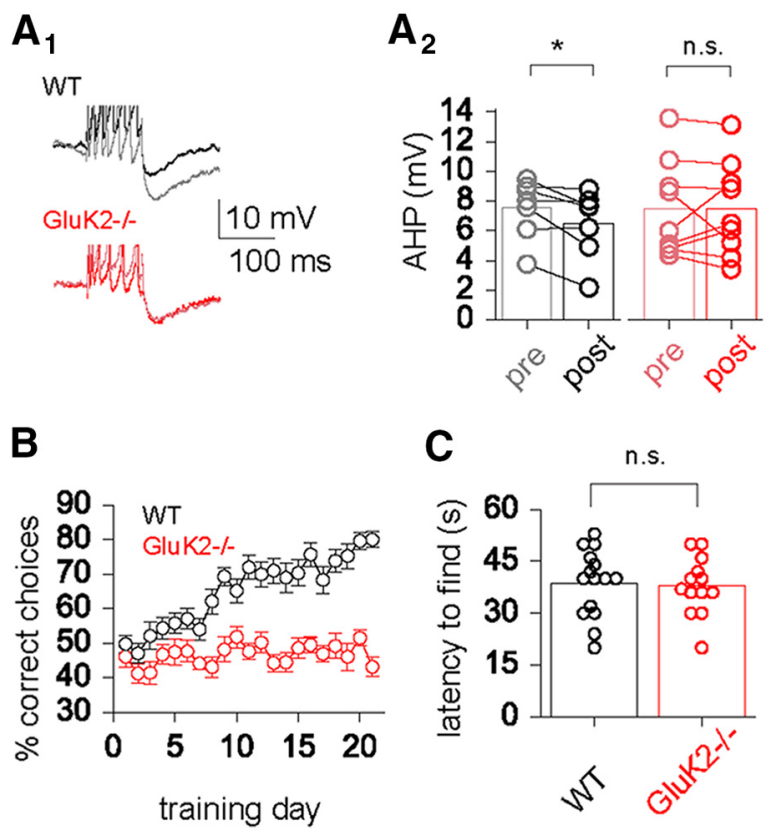

Figure 3. GluK2 activation is mandatory for AHP reduction and complex olfactory learning. $\boldsymbol{A}_{1}$, Averaged typical traces of a neuron from GluK2 $^{-1-}$ mice (red) and wild-type littermates (black) before (light shade) and after (dark shade) kainate application. The AP peaks are clipped to facilitate the comparison of the postburst AHP. $\boldsymbol{A}_{\mathbf{2}}$, Direct comparison of the absolute AHP amplitudes before and $20 \mathrm{~min}$ after kainate application for GluK2 $^{-\prime-}$ mice (red) and wild-type littermates (black). B, While wild-type mice (black) show a gradual learning curve, resulting in acquisition of the complex OD task for most (22 of 23) of the subjects, GluK2 $2^{-1-}$ mice (red) performance mostly remains at the level of chance. Only 2 of 23 knock-out mice were able to learn the task. Average performance for each day was calculated for all 20 trials of the day. C, Swarmplot of the latency to find the cookie in the buried food task in wild types (black) and GluK2 ${ }^{-1-}$ mice (red). In this simple olfactory task, there is no difference between genotypes. Values represent the mean \pm SEM. ${ }^{*} p<$ 0.05 .

tency to find cookie: wild types, $38.7 \pm 2.2 \mathrm{~s}, n=15$; GluK2 $^{-1-}$ mice, $37.9 \pm 2.9 \mathrm{~s} ; n=13 ; p=0.82$; Fig. $3 C$ ), indicating that their decreased performance in the olfactory discrimination learning task cannot be attributed to a general olfactory impairment or to a lack of motivation.

Finally, we overexpressed GluK2 in piriform cortex layer II pyramidal neurons (Fig. 4A). Our prediction was that GluK2 overexpression will considerably enhance complex learning in the olfactory maze. Indeed, piriform cortex GluK2 injected rats $(n=19)$ showed a considerably faster learning curve, compared with control rats $(n=18$; Fig. $4 B$ ). Notably, the learning curves of the two groups began to separate on the second day of training (Fig. 4B1). Rats with piriform cortex overexpression of GluK2 reached the criterion for complex olfactory discrimination learning completion, $80 \%$ successes in the last 10 trials of the day, which is significantly faster than controls injected with GFP only (GluK2-injected group: $5.1 \pm 0.3$ training days, $n=19$; GFP only-injected control group: $6.9 \pm 0.3$ training days, $n=18 ; p<0.001$; Fig. 4B2). These genetic 
$A_{1}$

HIV1-CAMK2a-GLUK2-IRES-GFP

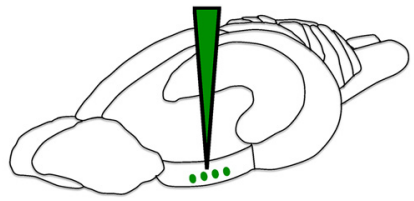

$B_{1}$

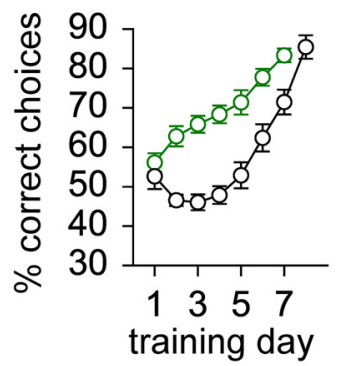

$\mathrm{A}_{2}$
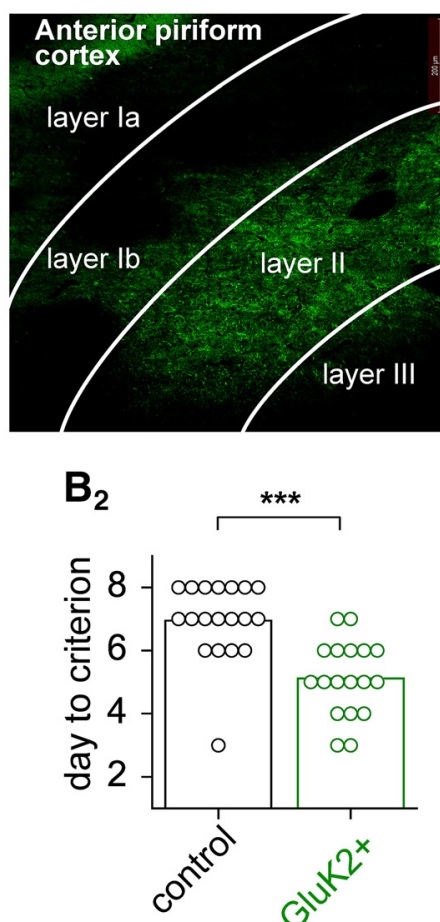

Figure 4. Enhanced complex olfactory learning is enhanced by GluK2 overexpression. $\boldsymbol{A}_{1}$, Schematic of virus injection in the rat piriform cortex. $\boldsymbol{A}_{2}$, Virus-infected pyramidal neurons in layer II indicated by GFP expression. Signal of GFP was enhanced using anti-GFP antibody. $\boldsymbol{B}_{\mathbf{1}}$, Time line of performance as a function of training day for GluK2-overexpressing rats (green) and controls injected with GFP virus only (black). To allow a more detailed look at the progress in performance for each day, the averaged percentage of correct choices for all 20 trials of the day are plotted. $\boldsymbol{B}_{\mathbf{2}}$, Swarmplot of the days needed to reach the criterion defined as a success rate of $>80 \%$ in the last 10 trials. GluK2-injected rats (green) complete complex olfactory discrimination learning significantly faster than the control rats (black). Values represent the mean \pm SEM. ${ }^{* * * *} p<0.001$.

modifications suggest that GluK2 receptors have a central role in complex olfactory learning.

\section{Discussion}

The first physiologic modification that is detected in single neurons during and after complex olfactory learning is enhanced intrinsic neuronal excitability by reduction of the slow AHP (Saar et al., 1998, 2002, 2012). We demonstrate in the piriform cortex that the synaptically activated GluK2-type glutamate receptor is the upstream mediator of this AHP reduction. Genetic manipulations (transgenic knock-out and region-specific virally mediated overexpression) demonstrate the necessity and sufficiency of GluK2-mediated intrinsic excitability changes for complex OD learning. We therefore provide a direct link between metaplasticity at the cellular level and complex learning at the behavioral level.

\section{Noncanonical signaling of GluK2 receptors and learning}

Previous studies in the hippocampus suggest that glutamatergic synaptic transmission reduces the AHP by noncanonical signaling of the GluK2 subtype glutamate receptor (Melyan et al., 2002, 2004; Fisahn et al., 2005; Grabauskas et al., 2007; Melyan and Wheal, 2011). In the piriform cortex, we provide a direct link between this cellular mechanism and complex olfactory learning. Com- plex olfactory learning occludes in vitro stimulation paradigms that result in AHP reduction in neurons from control animals (Fig. 1). Genetic knockout of GluK2 abolishes kainate-mediated reduction of the AHP and complex olfactory learning (Fig. 3). In addition, region-specific overexpression of GluK2 receptors enhances complex olfactory learning performance (Fig. 4). While noncanonical signaling of ionotropic glutamate receptors has been described, its functional relevance on a behavioral level is unclear (Valbuena and Lerma, 2016). This is the first direct demonstration that noncanonical signaling of ionotropic glutamate receptors has a selective impact on higher cognitive abilities.

\section{Long-lasting AHP reduction is induced and maintained via metabotropic activation}

Several studies indicate that the learning-induced reduction in neuronal adaptation and in AHP amplitude result from reduction in an acetylcholine-sensitive $\mathrm{Ca}^{2+}$ dependent potassium current (Sanchez-Andres and Alkon, 1991; Saar et al., 2001; Power et al., 2002). In particular, learning-induced enhanced excitability is mediated by long-term reduction in the conductance of the $\mathrm{sl}_{\mathrm{AHP}}$, in both the piriform cortex (Brosh et al., 2006) and hippocampus (Power et al., 2002). Olfactory discrimination learning-induced AHP reduction in the piriform is also PKC dependent (Seroussi et al., 2002) and ERK depen- 
dent (Cohen-Matsliah et al., 2007). Our data show that the same second-messenger systems are also required for activity-induced induction of the long-lasting AHP reduction. Moreover, synaptically induced and kainate-induced AHP reduction is occluded by prior complex learning; in vitro AHP reduction abolishes the difference in the average AHP amplitude between neurons from trained and control animals. Thus, our data support the hypothesis that glutamate secretion, which occurs during learning, induces long-lasting AHP reduction via a metabotropic mechanism. It should be noted that a possibility for AHP "contamination" by $\mathrm{GABA}_{\mathrm{B}}$ receptor-mediated synaptic inhibition cannot be completely ruled out. However, such a scenario is highly unlikely; OD learning-induced postburst AHP reduction is mediated by a decline in the conductance of the current that mediated the AHP (Saar et al., 2001), whereas synaptic inhibition is enhanced after learning (Kfir et al., 2014). Moreover, intracellular application of the calcium chelator abolished the differences in the AHP amplitude between neurons from trained animals and controls (Saar et al., 2001). This evidence implies that the OD learning-induced AHP reduction is induced and maintained by modulation of intrinsic membrane currents.

\section{Functional impact of global increases in intrinsic excitability}

Widely spread AHP reduction, and the resulting enhancement of neuronal excitability, is an ideal candidate for preparing neuronal networks for subsequent learning (Chandra and Barkai, 2018). This global unspecific upregulation of the sensitivity for encoding content has to be distinguished from the synapse-specific encoding of content. Several lines of evidence support the notion that an increase in intrinsic excitability triggered by the GluK2mediated AHP reduction is an ideal cellular mechanism for complex learning; one prerequisite for a metaplastic mechanism that primes a neuronal network for learning is that the metaplastic state change needs to last longer than its induction. This temporal delay criterion is met by GluK2-mediated AHP reduction. Our in vitro experiments as well as the aforementioned studies in the hippocampus demonstrate that the GluK2-mediated AHP reduction is long lasting. This is in contrast to the previously reported neuromodulatory AHP reduction mediated, for example, by noradrenergic stimulation of $\beta 1$ receptors (Madison and Nicoll, 1986). Importantly, learning-induced postburst AHP reduction in the piriform cortex is the first cellular modulation that is detected after complex OD learning; it is induced $2 \mathrm{~d}$ before enhanced synaptic connectivity appears (Saar et al., 1998, 2012; Knafo et al., 2005; Ghosh et al., 2015).

Changes in intrinsic excitability have been linked to plasticity and learning before. During auditory fear conditioning, neurons with enhanced intrinsic excitability are preferably recruited into new memory traces (GoutyColomer et al., 2016). Moreover, activity-induced synaptic strengthening also occurs in more excitable neurons. LTP is more readily induced when the AHP is reduced (Sah and Bekkers, 1996; Norris et al., 1998). Also, following complex olfactory discrimination learning the postburst
AHP is reduced and neuronal excitability is transiently enhanced in CA1 pyramidal neurons for $3 \mathrm{~d}$. During this period, the rats show enhanced learning capability in a different hippocampus-dependent task, the Morris water maze (Zelcer et al., 2006).

\section{Complex olfactory discrimination learning and rule learning}

Previously, we demonstrated complex olfactory learning results in the general enhancement of learning capabilities and thus can be described as rule learning ("learning how to learn," or "rule learning"). By acquiring an abstract set of rules when they reach the training criterion the first time, the rats reach the criterion much faster when presented with a different set of odors in the same task. Long-lasting AHP reduction in the pifirorm cortex is a prerequisite for rule learning (Saar et al., 1998; Zelcer et al., 2006; Chandra and Barkai, 2018). While we did not demonstrate the involvement of GLUK2 in rule learning, it is tempting to speculate that the GLUK2mediated reduction of the AHP during complex OD learning is the mechanism underlying the AHP reduction necessary for rule learning.

To conclude, our data suggest that GluK2-mediated AHP reduction, and the subsequent enhancement in neuronal excitability within the piriform cortex, is the mechanism that enables neuronal ensembles to enter into a state of metaplasticity at the cell and network levels that constitutes the mechanistic basis of complex OD learning. It will be of interest whether other brain areas, such as for example the hippocampus, are not only equally involved in this kind of complex learning, but also use similar molecular and cellular mechanisms. Interestingly, aberrant metaplasticity has been linked to cognitive dysfunction (Hulme et al., 2013). Indeed, GluK2 loss in humans results in intellectual disability (Motazacker et al., 2007). Our study is the first to provide a mechanistic hypothesis of how the loss of GluK2 in humans can result in intellectual disabilities by impairment of the AHP-related metaplasticity underlying complex learning and eventually rule learning. Modulation of this specific pathway suggests new avenues for the treatment of cognitive dysfunction as well as cognitive enhancement.

\section{References}

Abraham WC (2008) Metaplasticity: tuning synapses and networks for plasticity. Nat Rev Neurosci 9:387. CrossRef Medline

Abraham WC, Bear MF (1996) Metaplasticity: the plasticity of synaptic plasticity. Trends Neurosci 19:126-30. Medline

Brosh I, Rosenblum K, Barkai E (2006) Learning-induced reversal of the effect of noradrenalin on the postburst AHP. J Neurophysiol 96:1728-1733. CrossRef Medline

Chandra N, Barkai E (2018) A non-synaptic mechanism of complex learning: modulation of intrinsic neuronal excitability. Neurobiol Learn Mem 154:30-36. CrossRef Medline

Castillo PE, Malenka RC, Nicoll RA (1997) Kainate receptors mediate a slow postsynaptic current in hippocampal CA3 neurons. Nature 388:182-186. CrossRef Medline

Chamberlain SE, Sadowski JH, Teles-Grilo Ruivo LM, Atherton LA, Mellor JR (2013) Long-term depression of synaptic kainate receptors reduces excitability by relieving inhibition of the slow afterhyperpolarization. J Neurosci 33:9536-45. CrossRef Medline 
Cohen-Matsliah S, Brosh I, Rosenblum K, Barkai E (2007) A novel role for extracellular signal-regulated kinase in maintaining longterm memory-relevant excitability changes. J Neurosci 27:1258412589. CrossRef Medline

Constanti A, Sim JA (1987) Calcium-dependent potassium conductance in guinea-pig olfactory cortex neurones in vitro. J Physiol 387:173-194. CrossRef Medline

Gouty-Colomer LA, Hosseini B, Marcelo IM, Schreiber J, Slump DE, Yamaguchi S, Houweling AR, Jaarsma D, Elgersma Y, Kushner SA (2016) Arc expression identifies the lateral amygdala fear memory trace. Mol Psychiatry 21:364-75. CrossRef Medline

Fisahn A, Heinemann SF, McBain CJ (2005) The kainate receptor subunit GluR6 mediates metabotropic regulation of the slow and medium AHP currents in mouse hippocampal neurones. J Physiol 562:199-203. CrossRef Medline

Ghosh S, Reuveni I, Lamprecht R, Barkai E (2015) Persistent CaMKII activation mediates learning-induced long-lasting enhancement of synaptic inhibition. J Neurosci 35:128-39. CrossRef Medline

Grabauskas G, Lancaster B, O'connor V, Wheal HV (2007) Protein kinase signalling requirements for metabotropic action of kainate receptors in rat CA1 pyramidal neurones. J Physiol 579:363-373. CrossRef

Hasselmo ME, Stern CE (2018) A network model of behavioural performance in a rule learning task. Philos Trans $\mathrm{R}$ Soc Lond B Biol Sci 373:20170275. CrossRef Medline

Hulme SR, Jones OD, Abraham WC (2013) Emerging roles of metaplasticity in behaviour and disease. Trends Neurosci 36:353-62. CrossRef Medline

Knafo S, Libersat F, Barkai E (2005) Dynamics of learning-induced spine redistribution along dendrites of pyramidal neurons. Eur $\mathrm{J}$ Neurosci 21:927-35. CrossRef Medline

Kfir A, Ohad-Giwnewer N, Saar D, Golomb D, Barkai E (2014) Learning-induced modulation of the GABAB-mediated inhibitory synaptic transmission. J Neurophysiol 111:2029-2038. CrossRef Medline

Louboutin JP, Strayer DS (2013) Relationship between the chemokine receptor CCR5 and microglia in neurological disorders: consequences of targeting CCR5 on neuroinflammation, neuronal death and regeneration in a model of epilepsy. CNS Neurol Disord Drug Targets 12:815-829. CrossRef Medline

Madison DV, Nicoll RA (1984) Voltage clamp analysis of cholinergic action in the hippocampus. J Physiol 354:319-331.

Madison DV, Nicoll RA (1986) Actions of noradrenaline recorded intracellularly in rat hippocampal CA1 pyramidal neurones, in vitro. J Physiol 372:221-244. CrossRef Medline

Melyan Z, Wheal HV (2011) Metabotropic actions of kainate receptors in the regulation of I(SAHP) and excitability in CA1 pyramidal cells. Adv Exp Med Biol 717:49-58. CrossRef Medline

Melyan Z, Wheal HV, Lancaster B (2002) Metabotropic-mediated kainate receptor regulation of IsAHP and excitability in pyramidal cells. Neuron 34:107-114. CrossRef Medline

Melyan Z, Lancaster B, Wheal HV (2004) Metabotropic regulation of intrinsic excitability by synaptic activation of kainate receptors. $J$ Neurosci 24:4530-4534. CrossRef Medline

Motanis H, Maroun M, Barkai E (2014) Learning-induced bidirectional plasticity of intrinsic neuronal excitability reflects the valence of the outcome. Cereb Cortex 24:1075-1087. CrossRef Medline

Motazacker MM, Rost BR, Hucho T, Garshasbi M, Kahrizi K, Ullmann R, Abedini SS, Nieh SE, Amini SH, Goswami C, Tzschach A, Jensen LR, Schmitz D, Ropers HH, Najmabadi H, Kuss AW (2007) A defect in the ionotropic glutamate receptor 6 gene (GRIK2) is associated with autosomal recessive mental retardation. Am J Hum Genet 81:792-798. CrossRef Medline

Moyer JR, Thompson LT, Disterhoft JF (1996) Trace eyeblink conditioning increases CA1 excitability in a transient and learning specific manner. J Neurosci 16:5536-5546. CrossRef Medline

Norris CM, Halpain S, Foster TC (1998) Reversal of age-related alterations in synaptic plasticity by blockade of L-type $\mathrm{Ca}^{2+}$ channels. J Neurosci 18:3171-3179. CrossRef Medline
Oh MM, Kuo AG, Wu WW, Sametsky EA, Disterhoft JF (2003) Watermaze learning enhances excitability of CA1 pyramidal neurons. J Neurophysiol 90:2171-2179. CrossRef Medline

Petrovic MM, Viana da Silva S, Clement JP, Vyklicky L, Mulle C, González-González IM, Henley JM (2017) Metabotropic action of postsynaptic kainate receptors triggers hippocampal long-term potentiation. Nat Neurosci 20:529-539. CrossRef Medline

Power JM, Wu WW, Sametsky E, Oh MM, Disterhoft JF (2002) Age-related enhancement of the slow outward calcium-activated potassium current in hippocampal CA1 pyramidal neurons in vitro. J. Neurosci 22:7234-7243. CrossRef Medline

Ramezani A, Hawley RG (2002) Overview of the HIV-1 lentiviral vector system. Curr Protoc Mol Biol Chapter 16:Unit 16.21. CrossRef Medline

Ruiz A, Sachidhanandam S, Utvik JK, Coussen F, Mulle C (2005) Distinct subunits in heteromeric kainate receptors mediate ionotropic and metabotropic function at hippocampal mossy fiber synapses. J Neurosci 25:11710-11718. CrossRef Medline

Saar D, Barkai E (2003) Long-term modifications in intrinsic neuronal properties and rule learning in rats. Eur J Neurosci 17:2727-2734. CrossRef Medline

Saar D, Grossman Y, Barkai E (1998) Reduced after-hyperpolarization in rat piriform cortex pyramidal neurons is associated with increased learning capability during operant conditioning. Eur J Neurosci 10:1518-1523. CrossRef Medline

Saar D, Grossman Y, Barkai E (2001) Long lasting cholinergic modulation underlies rule learning in rats. J Neurosci 21:1385-1392. CrossRef Medline

Saar D, Grossman Y, Barkai E (2002) Learning-induced enhancement of post-synaptic potentials in pyramidal neurons. J Neurophysiol 87:2358-2363. CrossRef Medline

Saar D, Reuveni I, Barkai E (2012) Mechanisms underlying rule learning-induced enhancement of excitatory and inhibitory synaptic transmission. J Neurophysiol 107:1222-1229. CrossRef Medline

Sah P, Bekkers JM (1996) Apical dendritic location of slow afterhyperpolarization current in hippocampal pyramidal neurons: implications for the integration of long-term potentiation. $\mathrm{J}$ Neurosci 16:4537-4542. CrossRef Medline

Sanchez-Andres JV, Alkon DL (1991) Voltage-clamp analysis of the effects of classical conditioning on the hippocampus. J Neurophysiol 65:796-807. CrossRef Medline

Seroussi Y, Brosh I, Barkai E (2002) Learning-induced reduction in post-burst after-hyperpolarization (AHP) is mediated by activation of PKC. Eur J Neurosci 16:965-969. Medline

Telfeian AE, Federoff HJ, Leone P, During MJ, Williamson A (2000) Overexpression of GluR6 in rat hippocampus produces seizures and spontaneous nonsynaptic bursting in vitro. Neurobiol Dis 7:362-374. CrossRef Medline

Thompson LT, Moyer JR, Disterhoft JF (1996) Transient changes in excitability of Rabbit CA3 neurons with a time course appropriate to support memory consolidation. J Neurophysiol 76:1836-1849. CrossRef Medline

Valbuena S, Lerma J (2016) Non-canonical signaling, the hidden life of ligand-gated ion channels. Neuron 92:316-329. CrossRef Medline

Vignes M, Collingridge GL (1997) The synaptic activation of kainate receptors. Nature 388:179-182. CrossRef Medline

Xu X, Sun R, Jin R (2008) The effect of the ketogenic diet on hippocampal GluR5 and GluR6 mRNA expression and Q/R site editing in the kainate-induced epilepsy model. Epilepsy Behav 13:445-448. CrossRef Medline

Yang M, Crawley JN (2009) Simple behavioral assessment of mouse olfaction. Curr Protoc Neurosci Chapter 8:Unit 8.24. CrossRef Medline

Zelcer I, Cohen H, Richter-Levin G, Lebiosn T, Grossberger T, Barkai E (2006) A cellular correlate of Learning-induced metaplasticity in the hippocampus. Cereb Cortex 16:460-468. CrossRef Medline 